\title{
A NEW INEQUALITY FOR THE GREEN'S FUNCTION
}

\author{
WILLIAM C. FOX
}

1. Introduction. This note contains two new results on the Green's function of certain planar domains. One is concerned with the Green's function's boundary values, the other is an upper bound for the value at the pole of the "regular part" of the Green's function, an upper bound which is independent of the location of the pole. The significance of these results is indicated in their consequences, mainly (3.3) that the Green's function vanishes on any nondegenerate boundary component, and (3.4) an inequality which establishes the "best possible" value for Koebe's constant. The proofs are entirely elementary in character aside from use of the monodromy theorem, which is essential in dealing with simply connected domains. The vanishing of the Green's function on the boundary serves to establish that the Riemann mapping function is onto and thereby provides a reasonably short version of Riemann's original argument.

2. Preliminaries. If $D$ is a domain in any Riemann surface and if $d$ is a point of $D$, then a Green's function for $D$ with pole at $d$ is a potential function, $u$, harmonic and non-negative on the complement of $d$, such that

$$
u(C(z))+\ln \left|C^{-1}(z)\right|
$$

(the so-called regular part of $u$ ) is harmonic at $z=0$ if $c$ is any local coordinate mapping a neighborhood of $z=0$ onto a neighborhood of $d$, and such that $u \leqq v$ for every other such function $v$. An important fact about Green's functions, which is used frequently in the next section, is:

THEOREм 2.1. If $f$ is an analytic nonconstant map of the Riemann surface $A$ into the Riemann surface $B$ and if $V$ is a Green's function for $B$ with pole at $f(a)$ then $A$ has a Green's function $U$ with pole at $a$ and

$$
0 \leqq U \leqq V(f)
$$

on $A$.

This fact was first stated and proved by Poincaré [1] although it is contained implicitly in a much earlier paper [2]. A proof consists in approximating $A$ by a monotone sequence of domains with compact closure and sectionally analytic boundaries on each of which the

Received by the editors November 25, 1958. 
Dirichlet problem is solvable since the closure of each is the union of the closures of finitely many "disks" on which the Dirichlet problem is solvable, whence it is solvable on the domain itself by the theorem of H. A. Schwarz [3, Volume II] (that the union of two solvable domains whose boundaries meet in a finite set is itself solvable). Schwarz also proved that solvable domains of this sort admit Green's functions, again using his famous "alternating method." The resulting increasing sequence of Green's functions is dominated by $V(f)$, and so converges (to the desired $U$ ) by Harnack's theorem.

Schwarz was also, apparently, the first to recognize that the Poisson integral solves the Dirichlet problem for any disk and hence for any 1-1 conformal image of a disk. Thus, recorded here for reference is the following result.

LeMma 2.2. The complement in the sphere of any linear segment or of any disk admits a Green's function which vanishes continuously on the boundary.

This fact can, of course, be proved by elementary means without the use of Riemann's mapping theorem. Another fact recorded here for reference is

Leмma 2.3. If $f$ is a 1-1 analytic map of the Riemann surface $A$ onto the Riemann surface $B$, and if $V$ is a Green's function for $B$ with pole at $f(a)$, then $V(f)$ is a Green's function for $A$ with pole at a.

A final preliminary result generalizes the usual procedures for building single valued inverse branches of many-to-one mappings.

Lemma 2.4. Let $f$ be a meromorphic function mapping the domain $A$ onto $B$ and let $D$ be a simply connected subdomain of $B$. If $f$ is locally 1-1 and if $a_{1}, a_{2}, \cdots, a_{n}, \cdots$ are the points in $A$ for which $f\left(a_{i}\right)=d$ in $D$, then there exist 1-1 meromorphic functions $g_{1}, g_{2}, \cdots, g_{n}, \cdots$ mapping $D$ into $A$ such that $g_{i}(d)=a_{i}$ and $f\left(g_{i}(z)\right)=z$ for each $z$ in $D$. Moreover $g_{i}(D)$ is disjoint from the $g_{j}$-image of some neighborhood of $d, i \neq j$.

Proof. Let $g_{i}$ denote the inverse of $f$-restricted-to-a-neighborhoodof- $a_{i}$. Since the values of $f$ cover $D$ and $f$ is locally 1-1, it follows that $g_{i}$ can be extended along every path in $D$ emanating from $d$. By the monodromy theorem, $g_{i}$ has a meromorphic extension to all of $D$. Since $f\left(g_{i}(z)\right)=z$, it follows that $g_{i}$ is $1-1$. That the range of $g_{i}$ is disjoint from the $g_{j}$-image of some neighborhood of $d$ follows from the fact that $f$ is single valued. 
CoROlla Ry 2.5. If $A$ is any domain in the sphere which has a boundary component containing more than one point, then $A$ admits a 1-1 analytic map into the unit disk.

Proof. If $K$ is the nondegenerate boundary component of $A$, then $D$, the complement of $K$, is simply connected. Since $K$ contains at least two points, it is no restriction to suppose that these points are 0 and $\infty$. Thus, if $f(z)=z^{2}$ one can apply 2.4. In fact, any power function will do as well. So would the exponential function, or for that matter, the modular function used by Osgood [4] to reduce the Riemann mapping theorem from unbounded to bounded domains.

\section{3 . The main results.}

Lemma 3.1. (The PREliminary Inequality). The simple closed curve $J_{r}$ in the upper half plane which is mapped by the function $F_{r}(w)=w^{2}+r$ onto the circle whose radius is $r>0$ and whose center is the origin separates the sphere into two domains: $E_{r}$, the exterior one and $I_{r}$, the interior one. If $e$ is any point of $E_{r}$ then $E_{r}$ admits a Green's function $U_{r}$ (with pole at $e$ ) which is continuous on $\left(E_{r} \cup J_{r}\right)-(e)$ and vanishes on $J_{r}$. Moreover if $-e$ is not in $E_{r}$, then the inequality

$$
U_{r}(w) \leqq \ln \left|\frac{w+e}{w-e}\right|
$$

holds for all w in $E_{r}$.

Proof. By the methods of elementary differential geometry, one can check that for each point $j$ of $J_{r}$ there is at least one closed straight line segment $S_{j}$, of which $j$ is an end point, such that $S_{j}-(j) \subset I_{r}$. By 2.2 , the complement, $\left(E^{2}\right)^{-}-S_{j}$, of $S_{j}$ in the sphere admits a Green's function $V$ (with pole at $e \in E_{r}$ ) which is continuous on $\left(E^{2}\right)^{-}-(e)$ and vanishes on $S_{j}$. Since $\left(E^{2}\right)^{-}-S_{j} \supset E_{r}$, it follows by 2.1 that $E_{r}$ admits a Green's function $U_{r}$ with pole at $e$ in $E_{r}$ and that $0 \leqq U_{r} \leqq V$ on $E_{r}$. This implies that $U_{r}(w) \rightarrow 0$ as $w \rightarrow j\left(w\right.$ in $\left.E_{r}\right)$. Thus $U_{r}$ is continuous on $\left(E_{r} \cup J_{r}\right)-(e)$ and vanishes on $J_{r}$ as required.

Let the function $w=c(z)$ be defined by the relation

$$
w=e\left(\frac{1+z}{1-z}\right) .
$$

Its inverse, $z=c^{-1}(w)$, satisfies

$$
z_{-}^{*}=\frac{w-e}{w_{+}+e} .
$$


The function $c^{-1}(w)$ maps $E_{r}-[(e) \cup(-e)]$ into the plane punctured at $z=0$ so that $\ln \left|c^{-1}(w)\right|$ is harmonic on $E_{r}-[(e) \cup(-e)]$. Therefore, when $-e$ is $n o t$ in $E_{r}$, the function

$$
H_{r}(w)=U_{r}(w)+\ln \left|\frac{w-e}{w+e}\right|
$$

is harmonic on all of $E_{r}$ since, by definition of the Green's function, $H_{r}(c(z))=U_{r}(c(z))+\ln |z|$ is harmonic at $z=0$ when $c$ is any 1-1 conformal map of a neighborhood of $z=0$ onto a neighborhood, in $E_{r}$, of $w=e$. Noting that $U_{r}(w)=0$ on $J_{r}$, and that the harmonic function $H_{r}(w)$ is bounded by the greatest of its boundary values, one sees that

$$
H_{r}(w) \leqq M(r)=\operatorname{lub}_{w \in J_{r}}\left|\frac{w-e}{w+e}\right|
$$

for each $w$ in $E_{r}$.

Let $0<r^{\prime}<r$; because the curves $J_{r^{\prime}}$ shrink uniformly toward zero as $r^{\prime} \rightarrow 0$, it follows that

$$
\lim _{r^{\prime} \rightarrow 0} M\left(r^{\prime}\right)=\lim _{w \rightarrow 0}\left|\frac{w-e}{w+e}\right|=0
$$

so that $H_{r^{\prime}}(w) \rightarrow 0$ as $r^{\prime} \rightarrow 0$. Now $E_{r} \subset E_{r^{\prime}}$ so that, by definition of the Green's function, $H_{r} \leqq H_{r^{\prime}}$ on $E_{r}$. Thus

$$
H_{r}(w) \leqq \lim _{r^{\prime} \rightarrow 0} H_{r^{\prime}}(w)=0
$$

for each $w$ in $E_{r}$, which is the desired inequality.

Theorem 3.2. (The Final inequality). Let $D$ be a domain in the sphere with a boundary component $K$ which contains at least two different points, $p$ and $q$, let $d$ be a point of $D$, and let $T$ be the rational fractional transformation which sends $d, p, q$ respectively onto $0,1, \infty$. Then $D$ admits a Green's function $U$ with pole at $d$ such that $\lim U(t)=0$ as $t \rightarrow p(t$ in $D)$. Moreover if $H$ is defined by the rule

$$
H(z)=U\left(T^{-1}(z)\right)+\ln |z|
$$

then $H$ is harmonic at $z=T(d)=0$ and

$$
H(0) \leqq \ln 4
$$

Proof. It is convenient to write $T(K)=K^{*} T(D)=D^{*}$, etc. The complement, $C^{*}=\left(E^{2}\right)^{-}-K^{*}$, of $K^{*}$, in the sphere, is a simply connected domain (because $K^{*}$ is connected) containing $D^{*}$. In the notation of 3.1 with $r=1$ let $F_{1}(w)=w^{2}+1$ and let $L$ denote the open unit 
disk centered at the origin. The function $F_{1}$ maps $I_{1}$ onto $L$. By 2.4 , (since $C^{*}$ is simply connected and $F_{1}$ is locally $1-1$ on $E^{2}-(0)$ ), there are 1-1 analytic maps $g$ and $h$ of $C^{*}$ into $E^{2}-(0)$ with $g(0)=-i$ and $h(0)=i$. Not only does $h$ map $L$ onto $I_{1}$ but $g(L)$ must be disjoint from $h(L)=I_{1}$ since $g$ and $h$ are inverses of $F_{1}$ and $F_{1}$ is single valued on $I_{1}$. This implies that $g\left(C^{*}\right)$ is disjoint from $I_{1}$. Finally, because $F_{1}(0)=1$, one can say that $g(z) \rightarrow 0$ as $z \rightarrow 1\left(z\right.$ in $\left.C^{*}\right)$. Similarly for $h$.

The set $g\left(C^{*}\right)$ is contained in the complement of a disk (i.e., of a disk in the open set $I_{1}$ ) so that by 2.2 and 2.1 it follows that $C^{*}$, and $D^{*}$ also, admit Green's functions. Let $V$ be the Green's function for $E_{1}$ with pole at $e=g(0)=-i$ as given by 3.1 , and let $u$ be the Green's function of $D^{*}$ with pole at 0 . By 2.1 one can say that $u \leqq V(g)$ on $D^{*}$. As $z \rightarrow 1\left(z\right.$ in $\left.C^{*}\right), g(z) \rightarrow 0$ whence (since 0 is a boundary point of $E_{1}$ ) $V(g(z)) \rightarrow 0$ so that $u(z) \rightarrow 0$ as $z \rightarrow 1=T(p)\left(z\right.$ in $\left.D^{*}\right)$. The function $T$ is a 1-1 conformal map of $D$ onto $D^{*}$ and, by $2.3, U=u(T)$ is the Green's function of $D$ with pole at $d$, and $U(t)=u(T(t)) \rightarrow 0$ as $t \rightarrow p$, as required.

Since $H(z)=U\left(T^{-1}(z)\right)+\ln |z|=u(z)+\ln |z|$ is harmonic at $z=0$, and since $F_{1}(e)=0$, one sees that $H(0)=H\left(F_{1}(e)\right)$. By 2.1, one has $u(z) \leqq V(g(z))$, whence (since $g=F_{1}^{-1}$ ) by 3.1 ,

$$
u\left(F_{1}(w)\right) \leqq V(w) \leqq \ln \left|\frac{w+e}{w-e}\right|
$$

because in this case $e=-i$, and $-e=i$ is in $I_{1}$, not in $E_{1}$. Thus

$$
\begin{aligned}
H\left(F_{1}(w)\right) & =u\left(F_{1}(w)\right)+\ln \left|F_{1}(w)\right| \\
& \leqq \ln \left|\frac{w+e}{w-e}\right|+\ln \left|F_{1}(w)\right| \\
& \leqq \ln |w+e|+\ln \left|\frac{F_{1}(w)}{w-e}\right| .
\end{aligned}
$$

Because $F_{1}(e)=0$, the ratio $F_{1}(w) /(w-e)$ approaches the derivative of $F_{1}(w)=w^{2}+1($ at $w=e)$ as $w \rightarrow e$. It follows that

$$
H(0)=H\left(F_{1}(e)\right) \leqq \ln |2 e|+\ln |2 e|=\ln 4
$$

(since $|e|=1$ ) as required.

Corollary 3.3. If $D$ is a domain in the sphere and $K$ is a boundary component of $D$ which contains at least two points, then $D$ admits a Green's function (with pole at any arbitrary point, a, of $D$ ) which is continuous on $(D \cup K)-(a)$ and vanishes on $K$. 
This result is an immediate consequence of 3.2 , since $p$ (there) is an arbitrary point of $K$.

Riemann [5, pp. 1-42] was in effect the first to claim that the Green's function must vanish on the boundary of a simply connected surface bounded by a simple closed curve. However his proof (based on solving a boundary value problem) was defective, as Weierstrass had apparently [3, vol. II, pp. 65-83] recognized by 1863-1864. His student H. A. Schwarz gave the first sound proof [3, vol. II, pp. 133143] for simply connected plane domains with sectionally analytic boundary. Harnack [6, p. 120] claimed to extend this result to arbitrary simply connected domains but his proof was defective. Osgood [4] and [7] corrected Harnack's argument and Johansson [8] showed how the case of an arbitrary simply connected surface (with Green's function) can be reduced to the case treated by Osgood, concluding that the Green's function approaches zero along every divergent sequence of points. The approach taken here was suggested by a paper of Koebe's [9] in which he proved a weaker version of 3.1. In particular Koebe dealt only with simply connected domains and he failed to show that $H_{r} \leqq 0$; thus Koebe did not get the sharp result 3.2 which leads to the "best possible" value for Koebe's constant (e.g., 3.4).

Corollary 3.4 (Koebe's CONSTANT). If $F$ is 1-1 and analytic on the open unit disk $D$ then the largest disk centered at $F(0)$ and contained in $F(D)$ has a radius $r$ at least as great as $\left|F^{\prime}(0)\right| / 4$. In fact, $\left|F^{\prime}(0)\right| / 4$ $\leqq r|q| /(|q|-r) \leqq r$ for any boundary point $q$ of $F(D),|q| \neq v$.

Proof. It is no restriction to suppose that $F(0)=0$. Let $p$ be a boundary point of $F(D)$ such that $|p|=\operatorname{glb}\{|w|: w \in$ boundary of $F(D)\}$. By 2.3 , the function $U(w)=-\ln \left|F^{-1}(w)\right|$ is the Green's function of $F(D)$ with pole at $F(0)=0$. Let $T$ be the rational fractional transformation which sends $0, p, q$, respectively onto $0,1, \infty$, where $q$ is any boundary point of $F(D), q \neq p$. By 3.2, if $H$ is defined by the rule

$$
H(t)=U\left(T^{-1}(t)\right)+\ln |t|,
$$

where $t \in T(F(D))$, then $H(0) \leqq \ln 4$. But $H(t)=-\ln \left|F^{-1}\left(T^{-1}(t)\right)\right|$ $+\ln |t|$ and the ratio $F^{-1}\left(T^{-1}(t)\right) / t$ approaches the derivative (at $t=0)$ of $F^{-1}\left(T^{-1}\right)$ as $t \rightarrow 0$. Thus

$$
H(0)=+\ln \left|F^{\prime}(0) T^{\prime}(0)\right| \leqq \ln 4 .
$$

Now $T(w)=((p-q) / p)(w /(w-q))$ so that one checks the relation

$$
\left|T^{\prime}(0)\right|=\left|\frac{p-q}{p q}\right| \text {. }
$$


These facts lead to the conclusion that

$$
\frac{\left|F^{\prime}(0)\right|}{4} \leqq|p|\left|\frac{q}{p-q}\right| \text {. }
$$

Since $|p-q| \geqq|q|-|p|$, one can write

$$
\frac{\left|F^{\prime}(0)\right|}{4} \leqq|p|\left(\frac{|q|}{|q|-|p|}\right) \leqq|p|,
$$

as required.

The best possible value of Koebe's constant, i.e. the number $1 / 4$ in (3.4), is usually credited to Faber, as, e.g. in [10, p. 49] where it is derived from the theorem of Bieberbach (if $F$ is 1-1 and analytic on the open unit disk with $F(0)=0$ and $F^{\prime}(0)=1$ then $\left.\left|F^{\prime \prime}(0)\right| \leqq 2\right)$. The proof given here is noteworthy both for its geometric characteras opposed to the "hard analysis" required by others-and for the fact that it is based on Koebe's original method.

4. Riemann's proof of his mapping theorem. The fact that the Green's function of a simply connected plane domain vanishes continuously on the boundary, (3.3), is the key fact in carrying out the classical proofs of Riemann's mapping theorem. Given the existence criteria for the Green's function $u$ (2.5 and 2.1 show that having more than one boundary point is sufficient), one uses the fact that $h(z)$ $=U(z)+\ln |z-p|$ is harmonic at the point $p$ and so is, near $p$, the real part of a single valued analytic function $h(z)+i g(z)$. Since $h(z)$ is harmonic on the entire domain, so is its harmonic conjugate $g(z)$. Thus the harmonic conjugate of $u$ is just $v(z)=g(z)-\arg (z)$, whence

$$
F(z)=\exp (-(u(z)+i v(z)))
$$

is single valued and analytic on the domain. The vanishing of $u$ on the domain's boundary enables one to show that $F$ is onto the open unit disk. The analysis given by Osgood [11] of the level curves of $u$ shows that they are simple closed curves and that the directional derivative of $u$ in the direction outward from one of its level curves is positive because $u$ is smaller inside any one of its level curves than outside it. The net change in $v$ along a segment of level curve of $u$ is given by

$$
\int d v=\int\left(\frac{d u}{d n}\right)^{*} d s
$$

where $(d u / d n)^{*}$ is the derivative of $u$ in the outward normal direc- 
tion. Therefore, $v$ is strictly increasing as one moves around the level curve of $u$. Since $(u(z), v(z))$ are polar coordinates of $F(z)$, it follows that $F$ is $1-1$. This approach to Riemann's mapping theorem is certainly not new (indeed Riemann himself had in mind such an analysis) but it is nearly forgotten by the present generation, chiefly on account of the length and complexity of the classical accounts, and the dazzling brevity and elegance of the Fejér-Riesz proof.

\section{BIBLIOGRAPHY}

1. J. H. Poincare, Sur l'uniformisation des fonctions analytiques, Acta Math. vol. 31 (1907) pp. 1-63.

2. - Sur un thêorème de la thêorie générale des fonctions, Bull. Soc. Math France vol. 11 (1882-1883) pp. 112-125.

3. H. A. Schwarz, Gesammelte Mathematische Abhandlungen, Springer.

4. W. F. Osgood, On the existence of the Green's function for the most general simply connected plane region, Trans. Amer. Math. Soc. vol. 1 (1900) pp. 310-314.

5. B. Riemann, Gesammelte Mathematische Werke, Dover, 1953.

6. A. Harnack, Die Grundlagen der Theorie des Logarithmischen Potentiales und der Potentialfunction in der Ebene, Leipzig, 1887.

7. W. F. Osgood, Notes and errata: Volumes 1 and 2, Trans. Amer. Math. Soc. vol. 2 (1901) pp. 484-485.

8. S. Johansson, Ein Satz über die konforme Abbildung einfach zusammenhängender Riemannscher Flächen auf den Einheitskreis, Math. Ann. vol. 62 (1906) pp. 177-183.

9. R. Koebe, Über die Uniformisierung beliebiger analytischen Kurven, Göttingen, Nachrichten, 1907, pp. 191-210.

10. G. Valiron, Fonctions analytiques, Presses Universitaires de France, 1954.

11. W. F. Osgood, Lehrbuch der Functionen Theorie, Leipzig, 1907.

NORTHWESTERN UNIVERSITY 